\title{
A Scenario Tree based Stochastic Programming Approach for Multi-Stage Weapon Equipment Mix Production Planning in Defense Manufacturing
}

\author{
Xuan $\mathrm{Li}^{1}$, Yu Zhou ${ }^{2, a}$, Tianjun $\mathrm{Liao}^{3}$, Yajun $\mathrm{Hu}^{2}$ \\ ${ }^{1}$ Science and Technology on Information Systems Engineering Laboratory, National University of Defense \\ Technology, Changsha, 410073, P. R. China \\ ${ }^{2}$ School of Materiel Management and Safety Engineering, Air Force Engineering University, Xi'an 710051, P. R. \\ China \\ ${ }^{3}$ State Key Laboratory of Complex System Simulation, Beijing Institute of System Engineering, Beijing, 100101, P. R. \\ China
}

\begin{abstract}
The evolving military capability requirements (CRs) must be meted continuously by the multi-stage weapon equipment mix production planning (MWEMPP). Meanwhile, the CRs possess complex uncertainties with the variant military tasks in the whole planning horizon. The mean-value deterministic programming technique is difficult to deal with the multi-period and multi-level uncertain decision-making problem in MWEMPP. Therefore, a multi-stage stochastic programming approach is proposed to solve this problem. This approach first uses the scenario tree to quantitatively describe the bi-level uncertainty of the time and quantity of the CRs, and then build the whole off-line planning alternatives assembles for each possible scenario, at last the optimal planning alternative is selected on-line to flexibly encounter the real scenario in each period. A case is studied to validate the proposed approach. The results confirm that the proposed approach can better hedge against each scenario of the CRs than the traditional mean-value deterministic technique.
\end{abstract}

\section{Introduction}

The multi-stage weapon equipment mix production planning (MWEMPP) is crucial to maintain the military power of one country and has the properties of high-risk, multi-period and tremendous investment [1]. Therefore, the MWEMPP would be particularly important in the national defence industry. However, the combat capability requirements evolve with the ever more demanding mission tasks. Meanwhile, the exact values of the evolving CRs are unknown. That is, each CR has two uncertain parameters, i.e. time and quantity, and they are evolving with the period. The possible value ranges of the time and quantity can be predicted, but the corresponding distributions are unknown in each period. Thus, MWEMPP contains a multistage programming problem under the uncertain CRs.

There are some methods for the uncertain multistage programming problem, such as dynamic programming [2] and markov decision process [3]. But they need strict conditions on the probability of the event and the relations of the variables in each period. With the continuous increase of

\footnotetext{
${ }^{a}$ Corresponding author : zhouyu_gfkd@126.com
} 
computing power, the stochastic programing with multilayer scenario tree is more and more popular to model the multistage uncertain problem [4-6]. The multilayer scenario tree can model the arbitrary stochastic evolutions of the uncertain parameters as a group of discrete scenarios without the need to know the detailed probability distribution. Moreover, there is no limitation for the number of the uncertain parameters. Hence, the uncertain parameters of the CRs can be easily interpreted as a scenario tree. Furthermore, the stochastic programming method can adjust the development time and the Development budget to hedge against the various scenarios in each period.

Based on these surveys, we seek to propose a scenario tree based stochastic programming approach to model and solve MWEMPP problem. First, the multilayer scenario tree is built to model the evolution of the uncertain CRs. Hence, a specific computational process is proposed for the scenario tree generation. Finally, a multi-stage stochastic programming model is developed to solve effectively solve MWEMPP problem. A case is provided to illustrate the advantages of the proposed stochastic programming approach. To the best of our knowledge, this approach has not been proposed in other fields, and it is first proposed here.

\section{Problem statement}

Suppose that there are $C$ CRs and $M$ weapon categories, in which each CR is supported by more than one weapon category and vice versa. Each weapon category contains a number of weapon models. For each weapon model, the capability values depend on the corresponding development time and Development budget. In each weapon category, the weapon models have different capability values when they have equivalent development time and Development budget. The weapons development processes are shown in Fig. 1.

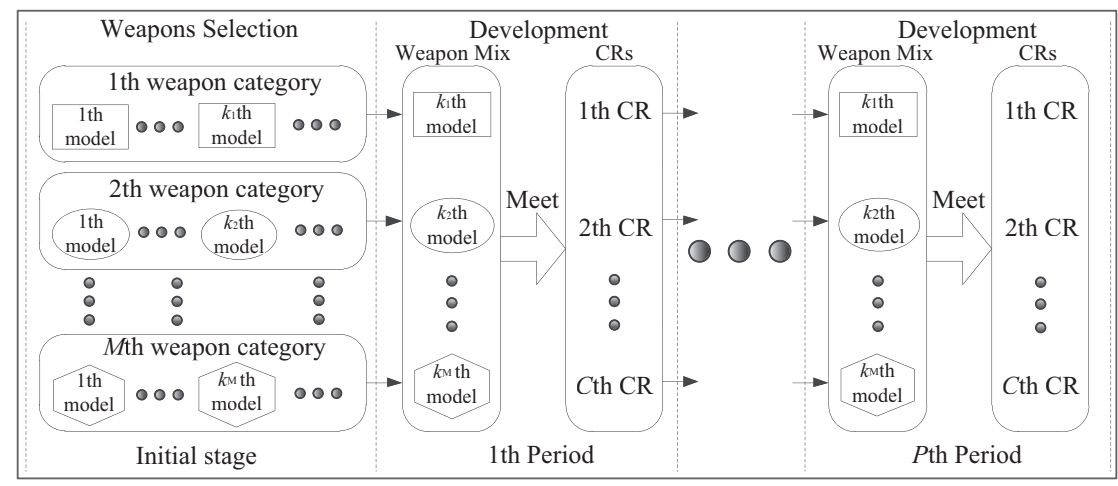

Figure 1. The processes of MWEMPP

As shown in Fig. 1, the $M$ weapon models must be selected from each category at the initial stage to form a weapon mix. Then the weapon mix is produced to meet the evolving CRs continuously in each period. Therefore, the corresponding weapon equipment mix contains two kinds of optimizations, one of which is to determine the weapon mix and the other one is to determine the development time and Development budget in each period. The objectives are to minimize the capability gaps between the weapon mix and the CRs in each period. In this context, the capability deficiencies and surpluses between weapon mix and the CRs are all recognized as the capability gaps.

\section{The multistage stochastic programming approach}

\subsection{Scenario tree modeling for the uncertain CRs}


We begin to use the scenario tree to model the uncertain CRs based on the formulations of them. All the possible evolutionary processes of CRs can be modeled by a scenario tree, as shown in Fig. 2.

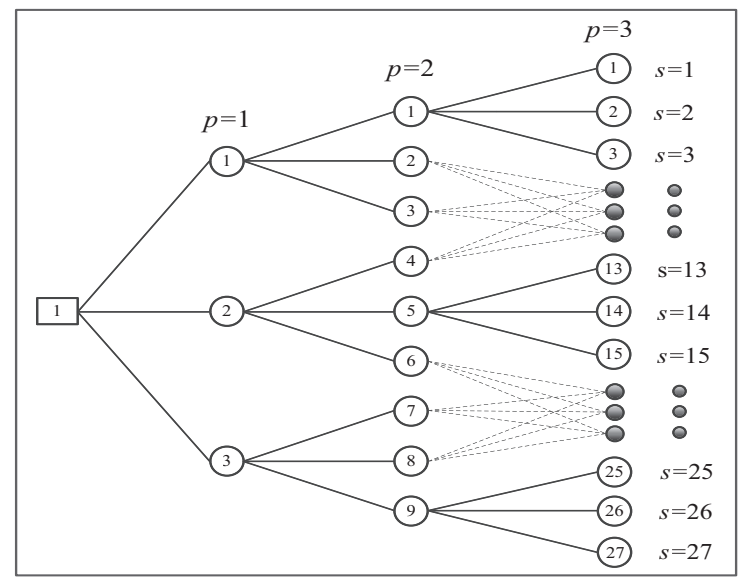

Figure 2. An example of a 3-periods scenario tree

Each circular node represents one possible state of the CRs corresponding to previous period. The path from the root node (rectangular node) to a leaf node (circular node) is called a scenario, i.e., a possible evolutionary process of the CRs over the entire planning horizon. For instance, the fifteenth scenario consists of the second node in the first period, the fifth node in the second period and the fifteenth node in the third period. Thus, the uncertain CRs can be converted into a number of certain scenarios in a scenario tree. Furthermore, the amount of CRs is assumed to be up to 5 kinds in the context of defense manufacturing, so that the scenario tree is generated in a manageable size.

\subsection{Scenario tree generation}

It is possible to obtain the time requirement interval $\left[R t l_{p, c}, R t u_{p, c}\right]$ and the quantity requirement interval $\left[R q l_{p, c}, R_{q u p_{e, c}}\right]$ of the CRs in each period, but the possible values of them are not available due to the confidential causes. Therefore, we propose a practical approach combining Monte Carlo simulation to build the scenario tree. The process of this approach is presented as follows:

Step 1: Determine the branch $\mathrm{Zp}$, the time requirement interval $\left[R t l_{p, c}, R t u_{p, c}\right]$ and the quantity requirement interval $\left[R q l_{p, c}, R q u_{p, c}\right]$ in each period.

Step 2: Divide the intervals of time requirement and quantity requirement into $Z p$ parts, respectively.

Step 3: Initialize $p=0$ and $z_{p}=0$. Select one part randomly out from the $Z_{p}$ parts of the time requirement without replacement, then within which generate an integer number randomly for $R t_{p, z, c}$ in this part.

Step 4: Select one part randomly out from the $Z_{p}$ parts of the quantity requirement without replacement, then within which generate a real number randomly for $R q_{p, z, c}$ in this part.

Step 5: Set $z_{p}=z_{p}+1$, repeat steps 3 4 until $z_{p}=Z_{p}$.

Step 6: Set $p=p+1$, repeat steps $2 \sim 5$ until $p=P$.

Step 7: Use the $\sum_{p=1}^{P} Z_{p}$

group values of the CRs to generate the scenario tree.

\subsection{Multistage stochastic programming model}

For the multi-period uncertain production planning problem, the traditional method is the mean-value deterministic programming [7]. This technique optimizes a group of fixed variables to cope with each 
possibility of the CRs. Contrary to the MVDP model, the multistage stochastic programming (MSP) model generates different variables in each node to hedge against the variations of the CRs. To build the weapons multistage stochastic programming model, the constraints of "non-anticipativity" have to be considered firstly [8]. Hence, the MSP model is formulated as follows:

$$
\begin{array}{ll}
\min & \sum_{p=1}^{P} \sum_{n_{p}=1}^{N_{p}} \pi\left(n_{p}\right) \times\left(\sum_{c=1}^{C}\left(w_{p, n, c}^{+} d_{p, n, c}^{+}+w_{p, n, c}^{-} d_{p, n, c}^{-}\right)\right) \\
\text {s.t. } & W C_{p, n, c}-d_{p, n, c}^{+}+d_{p, n, c}^{-}=R q_{p, z, c} \\
& d_{p, n, c}^{+}, \quad d_{p, n, c}^{-} \geq 0 \\
& z_{p}= \begin{cases}Z_{p} ; & \bmod \left(n_{p} / Z_{p}\right)=0 \\
\bmod \left(n_{p} / Z_{p}\right) ; & \bmod \left(n_{p} / Z_{p}\right) \neq 0\end{cases} \\
& N_{p}=\prod_{p^{\prime}=1}^{p} Z_{p^{\prime}} \quad P, N_{p}, Z_{p} \in ¥^{+}
\end{array}
$$

where $\pi\left(n_{p}\right)$ is considered as the unconditional probability to realize the possibility of the CRs in the $n_{p}$ th node, or the relative importance among the nodes in the same period. Constraint (2-4) means that the weapon mix generates distinctive capability value $W C_{p, n, c}$ to meet each possible value $R q_{p, z, c}$. Constraint (5) describes the relationship between $n_{p}$ and $z_{p}$. The $\bmod (*)$ represents the remainder operation.

\section{Case study}

\subsection{Problem description}

The C4ISR weapon equipment mix production planning [9] which is one important kind of MWEMPP problem is studied to validate the proposed hybrid approach. Thus, the C4ISR weapons need to be planned to satisfy multiple CRs of joint operations. Suppose that there are five weapon categories to be planned to meet seven CRs in the future. The data on the C4ISR weapons are show in Table 1.

Table 1: The data on the C4ISR weapons

\begin{tabular}{|c|c|c|c|c|c|c|c|c|c|}
\hline & NCWM & OPB & CR1 & CR2 & CR3 & CR4 & CR5 & CR6 & CR7 \\
\hline RSate & 5 & $0 \sim 300$ & $\sqrt{ }$ & $\sqrt{ }$ & $\sqrt{ }$ & $\sqrt{ }$ & & & \\
\hline AWACS & 4 & $0 \sim 200$ & $\sqrt{ }$ & $\sqrt{ }$ & & $\sqrt{ }$ & & & $\sqrt{ }$ \\
\hline MHAUAS & 6 & $0 \sim 100$ & $\sqrt{ }$ & $\sqrt{ }$ & $\sqrt{ }$ & & $\sqrt{ }$ & & \\
\hline BLOSDL & 3 & $0 \sim 80$ & & & & $\sqrt{ }$ & $\sqrt{ }$ & $\sqrt{ }$ & \\
\hline C2S & 3 & $0 \sim 100$ & & & & & $\sqrt{ }$ & $\sqrt{ }$ & $\sqrt{ }$ \\
\hline
\end{tabular}

All of the notations in Table 1 are listed in the following: (1) CR represents one capability requirement. (2) CR1 is the target identification. (3) CR2 is the location tracking. (4) CR3 is the constantly monitoring. (5) CR4 is the information transition and distribution. (6) CR5 is the interoperability. (7) CR6 is the self-adaption. (8) CR7 is the command and control. (9) NCWM is the numbers of the candidate weapon models in each C4ISR weapon category. (10) OPB is the optional development budget for each C4ISR weapon category. (11) RSate is the reconnaissance satellite. (12) AWACS is the airborne warning and control system. (13) MHAUAS is the medium to high altitude unmanned aerial system. (14) BLOSDL is the beyond line of sight data link. (15) C2S is the command and control system. 


\subsection{Validation of the MSP model}

We use the MVDP, MSP, ideal multi-stage programming (IMP) model to solve the addressed case, respectively, in order to find which one has more advantages to hedge against the uncertainties of the CRs, in order to investigate the degree that the MSP model diminishes the uncertainties of CRs. The respective global capability gap of the three models is shown in Fig. 3.

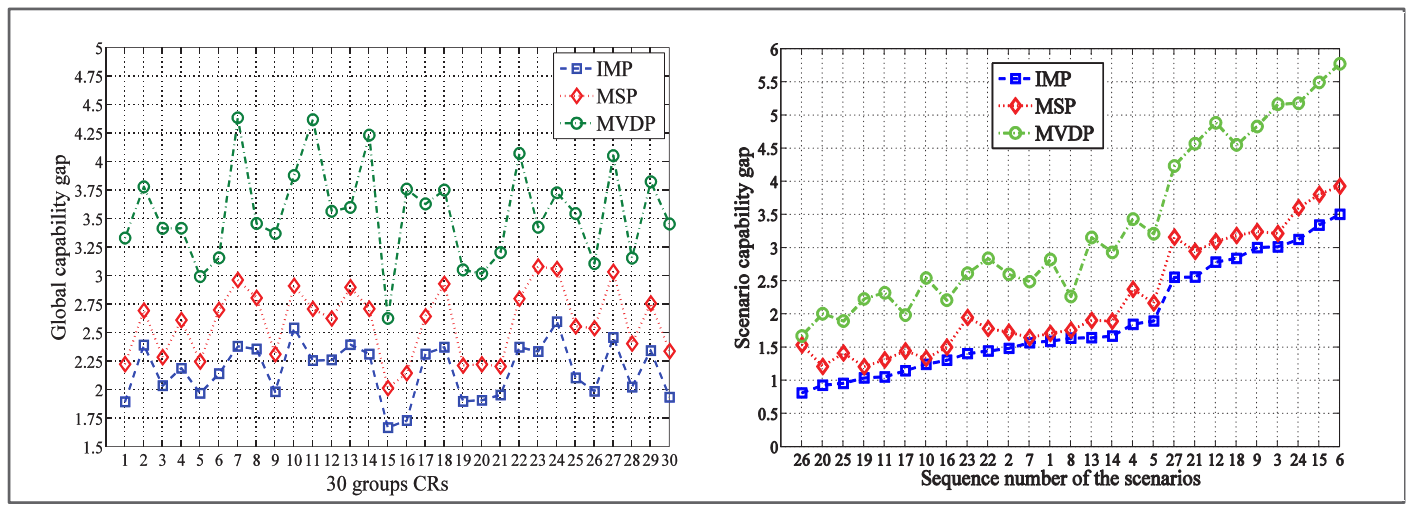

Figure 3. The capability gap of the three models

As shown in Fig. 3, for each group of CRs, IMP model gets the smallest global capability gap, and it represents the ideal values without considering the uncertainties of the CRs. The MSP model is closer to the ideal values than the MVDP model under each group CRs. This is because MSP takes different recourse actions to encounter each possible CRs, and MVDP model generates the fixed solutions to encounter all scenarios in each period. However, because of the constraints of the "nonanticipativity', the MSP model cannot offset the negative effects of uncertainties completely. Thus, it never achieves the level of the IMP model.

As shown of the scenario capability gap, the scenarios are sequenced by the values of the capability gaps generated by the IMP model in ascending order. Compared with the MVDP model, the MSP model is closer to the ideal capability gap curve, except individual scenario such as the 26th and the 23th. Furthermore, the extreme scenarios which are from the 27th to the 6th bring little influences to the close degree of the curves between the MSP model and the IMP model. In contrast, the distance of the curves between the MVDP model and the IMP model enlarges. It means that the MSP model have the better robustness than the MVDP model.

According to the above analysis, the MSP model outperforms the MVDP model, but there are no free lunches. Next, we change the quantity of the budget to test the variation of the global capability gap corresponding to the three models. The results are shown in Fig. 5.

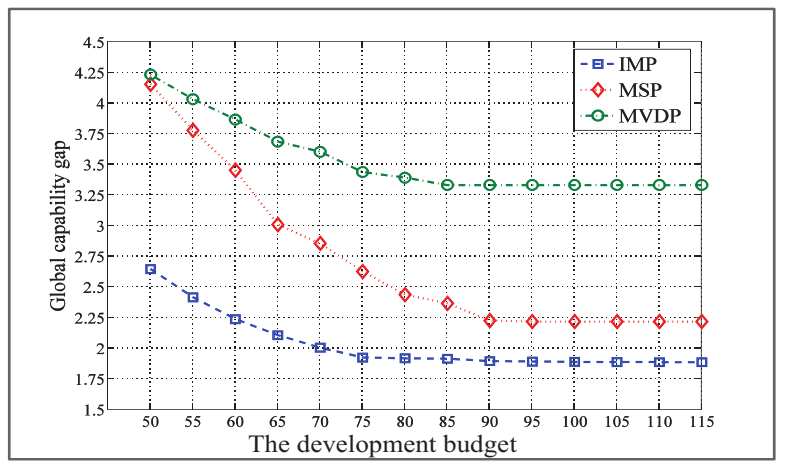

Figure 4. The variations of the global capability gap with the budget 
All of the three models obtain a smaller global capability gap with a larger budget. When the budget increases to a certain value, the gaps do not reduce any more. This is because the more budget are, the larger optional ranges of variables are. Thus, the alternative space contains better solutions. Furthermore, the gap of the MSP reduces more than MVDP model and IMP model when the budget increases. Obviously, only if the budget is more than 90 normalized amounts, the MSP model can obtain the optimal global capability gap which is close to the ideal value. This is because the MSP model can adjust the development time and the Development budget to avoid capability deficiency and surplus in different scenarios. When the capability requirements are large, the MSP model needs more budgets to adjust the development time and the Development budget. Thus, it naturally needs more budget.

\section{Conclusions}

We comparatively studied the MSP model with the traditional MVDP model and the ideal IMP model. The primary conclusions are as follows: (1) Both of the global capability gap and the scenario capability gap generated by the MSP model are smaller than those generated by the MVDP model. (2) The budget imposes a large influence to the MSP model. When the budget decreases, the MSP model obtains a larger global capability gap and closes to the MVDP model. When the budget increases, it derives a smaller global capability gap and closes to the IMP model. (3) The interval sizes of the CRs differs from each other in some extreme cases, thus, it means that we need to study the scenario tree with flexible branches to model the more widely evolution of the uncertain CRs.

\section{Funding}

This research is supported in part by the National Natural Science Foundation of China under contract Nos 71401167, 71571185.

\section{References}

1. Y. Zhou, J. Jiang, Z. Yang, Y. Tan. Proc IMchE Part B: J Engineering Manufacture, 2014.

2. Lew, A., \& Mauch, H. Berlin, Germany: Springer-Verlag, 2006.

3. Ferreira, K. D., \& Wu, D. D. Int. J. Development Economics, 134, 95-107, 2011.

4. Ahmed, S., \& Sahinidis, N. V. Operations Research, 51(3), 461-371, 2003.

5. Alfieri, A., \& Brandimarte, P. Germany: Springer-Verlag, 2005.

6. Heitsch, H., \& Römisch, W. Mathematical Programming, 118, 371-406, 2009.

7. Birge, J. R. New York, USA: Springer-Verlag Inc, 1997.

8. Ahmed, S., King, A. J., \& Parija, G. Journal of Global Optimization, 26(1), 3-24, 2003.

9. Y. Zhou, K. Yang, Y. Tan, Z. Yang. Proc. of the 7th SOSE, Genoa, Italy, 2012. 